\title{
EXCELENCIA ACADEMICA FAUA Y EXCELENCIA PROFESIONAL
}

Responsable: $\mathrm{Arq}^{\circ}$ Carlos Salamanca Oviedo

\section{RESUMEN}

La "Excelencia Profesional" como consecuencia de la "Excelencia Académica" es un tema de investigación de suma importancia en la formación universitaria de los estudiantes de la Facultad de Arquitectura, Urbanismo y Artes de la UNJBG.

Desarrollando la investigación se determinó que la excelencia académica está estrechamente ligada con la autoevaluación, la evaluación y la acreditación académica.

El presente trabajo de investigación trata de interiorizarse en el tema de la excelencia académica, pero en el proceso de desarrollo se ha encontrado que lo primero que se debe resolver es la autoevaluación, la evaluación y la acreditación de la FAUA; por lo tanto, se tuvo que profundizar esta temática arribando a conclusiones importantes. Por lo tanto, es imprescindible que las Facultades de Arquitectura de las Universidades Peruanas se sometan a este proceso y, más aún, la Facultad de Arquitectura, Urbanismo y Artes de la Universidad Nacional Jorge Basadre Grohmann, para alcanzar excelencia académica y, por ende, la excelencia profesional.

\section{ABSTRACT:}

The "Professional Excellency" as consequence of the "Academic Excellency" it is a topic of investigation of supreme importance in the university formation of the students of the College of Architecture, Urbanism and Arts of the UNJBG.

Developing the investigation was determined that the academic excellence is closely bound with the selfevaluation, the evaluation and the academic good repute. The present investigation work, is about to intern in the topic of the academic excellence but in the development process, it has been that the first thing that should be solved is the self-evaluation, the evaluation and the good repute of the FAUA, therefore one had to deepen this thematic one arriving to important conclusions.

Therefore it is indispensable that the Colleges of Architecture of the Peruvian Universities undergo this process and more even the College of Architecture, Urbanism and Arts of the National University Jorge Basadre Grohmann, to reach academic excellence and therefore the professional excellence.

\section{INTRODUCCIÓN}

La Universidad Nacional Jorge Basadre Grohmann y dentro de ella la Facultad de Arquitectura Urbanismo Artes no cumplen óptimamente los fines y objetivos que les corresponde como entes responsables de la educación superior de la juventud de la localidad y la región Tacna, tales son:

La Excelencia Académica, investigación cientifica, proyección y extensión social; por lo, tanto es necesario investigar las causas para que se revierta esta situación, asi como se planteen nuevos lineamientos académicos.

Los Fundamentos Teóricos, que nos permitirán analizar la situación real de la Facultad de Arquitectura, Urbanismo y Artes, se adhieren a los principios y valores institucionales establecidos en el Proyecto de Desarrollo Universitario de la Universidad Nacional Jorge Basadre Grohmann, planteados en el último Estatuto que la gobierna, aprobados por acuerdo de Asamblea Universitaria. Se orientan a:

Excelencia Universitaria. Nuestro que hacer debe desarrollarse con criterios de excelencia que permitan alcanzar y mantener elevados niveles de calidad. El aseguramiento de la calidad debe considerar procesos permanentes de auto evaluación por parte de cada uno de los estamentos que componen la FAUA y cuyo marco de referencia deben ser los estándares de medición de aceptación general.

Autonomía Universitaria. Los miembros de la comunidad universitaria de la UNJBG y, por ende, de la FAUA tienen la obligación y el derecho de decidir sobre el quehacer institucional de manera organizada y democrática.

Pluralismo Académico. Nuestra Universidad acepta las expresiones y manifestaciones de las distintas corriente del pensamiento universal dentro del marco de tolerancia y respeto.

Mutuo Respeto. La UNJBG y la FAUA garantizan el respeto a la diversidad y a la dignidad de TODOS SUS MIEMBROS.

Ética y Moral Profesional. El quehacer de la UNJBG y de la FAUA debe girar en torno a los más elevados principios éticos tendientes a dignificar la imagen institucional y la de sus miembros. Reconocemos nuestra responsabilidad con la sociedad, mediante la preservación del medio ambiente y aporte activo y permanente al desarrollo de nuestra región.

Actitud Crítica y Autocrítica. Somos una comunidad con conciencia crítica, proactiva y 
empática, lo cual se manifiesta en la capacidad de autocrítica, tolerancia y comprensión.

Libertad de Cátedra El docente tiene autonomía para plantear su metodología de enseñanza siempre enmarcada en la sumilla de la asignatura y los contenidos de acuerdo al perfil del profesional.

Finalmente, el objetivo general es fortalecer la calidad de las actividades académicas en función de estándares nacionales e internacionales en docencia e investigación, favoreciendo el liderazgo de la Universidad Nacional Jorge Basadre Grohmann y de la Facultad de Arquitectura, Urbanismo y Artes de la ciudad de Tacna, a nivel regional e internacional.

\section{MATERIALESYMÉTODOS}

Para la Investigación se ha efectuacio una revisión de la legislación internacional y nacional referida al tema, asi como propuestas de estándares para la acreditación en otras universidades de América y Europa.

Para la consecución de los resultados se han aplicado técnicas de investigación como los modelos de encuestas, entrevistas e investigación bibliográfica, y para recopilación de esta información se utilizaron instrumentos que a continuación se detallan:

\section{Información para Procesos de Autoevaluación Uso de Instrumentos \\ DOCENTES \\ Dimensión 1: \\ La Carrera en el Contexto Institucional}

1. La misión de la universidad está claramente formulada.

2. La misión de la Facultad concuerda con la de la Universidad

3. El servicio que brinda el personal administrativo es eficiente y eficaz.

4. El acceso al sistema de información de la Facultad es eficiente y eficaz.
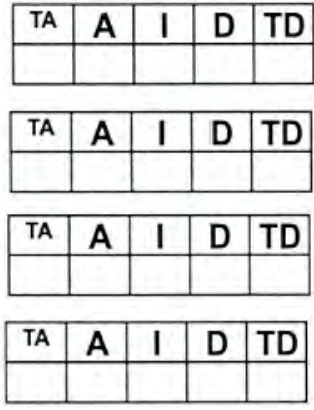

5. La Facultad cuenta con un sistema de comunicación eficiente y eficaz que facilita la coordinación entre sus distintas áreas y las de la Universidad.

6. Los directivos convocan, motivan y dan los lineamientos claros para lograr los objetivos de la Facultad.

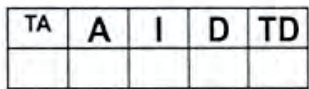

7. Los directivos dedican el tiempo necesario para el desempeño eficiente de su unidad.
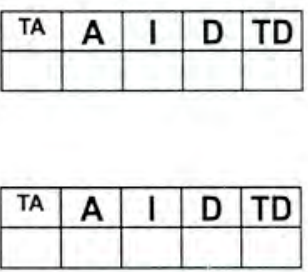

8. Conoce las normas de administración de la Facultad?

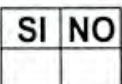

9. Las normas que emite la dirección de la Facultad están claramente definidas

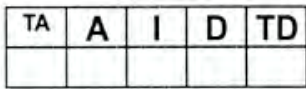

10. Las acciones de motivación estimulan al docente para contribuir al logro de los propósitos institucionales.

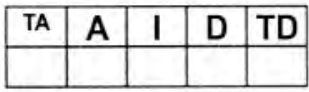

\section{Dimensión 2: Proyecto Académico}

1. El perfil del egresado está claramente formulado.

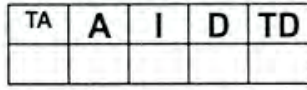

2. El número de personal administrativo es suficiente para apoyar su labor académica.

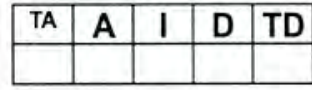

3. El número de docentes y personal administrativo es suficiente para atender las necesidades académicas y de servicio de la población estudiantil.

4. Su horario programado para tutoria toma en cuenta la carga académica de los alumnos.

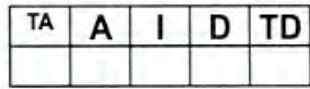

5. El proceso de graduación está claramente formulado.

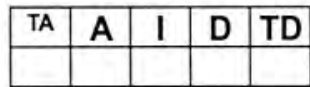

6. El proceso de titulación está claramente formulado.
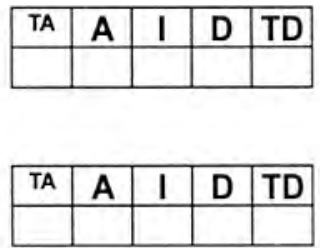

\section{Dimensión 3 : Docentes, Alumnos, Egresados}

1. El número de alumnos matriculados en su clase de teoría es el apropiado para garantizar el aprendizaje y tambien el número de docentes dedicados a la enseñanza de las clases. El número de alumnos matriculados en su clase de práctica es el apropiado para garantizar el aprendizaje.

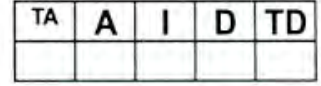


Dimensión 4: Infraestructura y Equipamiento

1. Las aulas de teoría presentan condiciones operativas apropiadas para el desarrollo de las clases Las prácticas se desarrollan en ambientes con condiciones operativas apropiadas

2. Cuenta con un ambiente apropiado para realizar sus funciones no lectivas
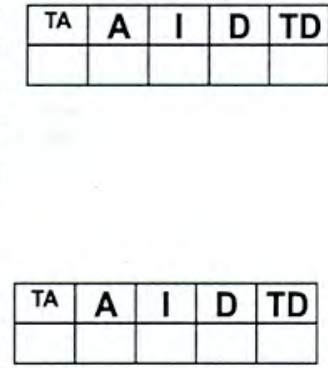

3. ¿Cuenta con equipos de informática disponibles para el desarrollo de sus funciones?

4. ¿Las computadoras a su disposición están conectadas en red?

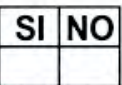

5. Cuenta con equipos de calidad (computadoras, fotocopias, impresoras, etc), para elaborar el material que emplea en sus clases

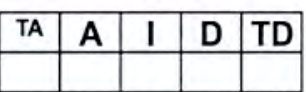

6. El servicio de atención de la salud cuenta con locales y equipamientos apropiados.

7. Encuentra en la biblioteca la información requerida o el apoyo para conseguirla.

8. Los préstamos internos e interbibliotecarios cubren los requerimientos que demanda su labor.

9. El servicio de reproducción de documentos en la Biblioteca es eficiente y eficaz.

10. El horario de atención de la biblioteca cubre los requerimientos que demanda su labor.

11. Encuentra en la Biblioteca información documentada (libros, revistas, videos, etc), actualizada y de calidad para su formación profesional y personal.
12. Los ambientes de laboratorio son suficientes en cantidad y calidad para el desarrollo de las actividades programadas.

13. El material que disponen los laboratorios es suficiente en cantidad y calidad para el desarrollo de las actividades programadas.

14. Los alumnos disponen de salas de informática debidamente equipadas para el desarrollo de sus actividades.

15. Se cuenta con el software requerido para el desarrollo de las actividades académicas.
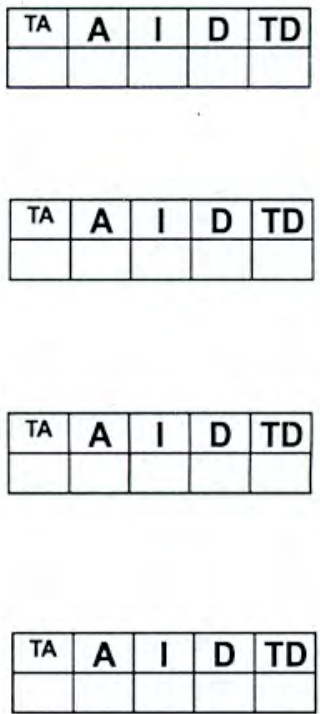
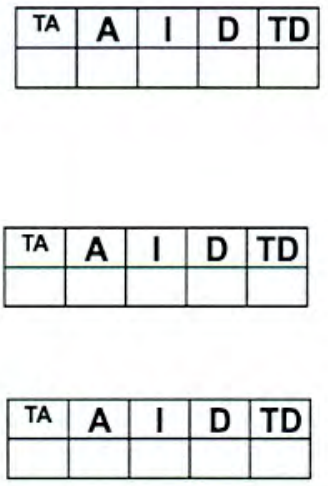

\section{Autoevaluación, uso de Instrumentos}

\section{Administrativos}

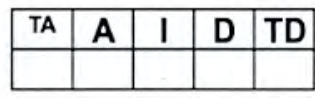
claramente formulada.

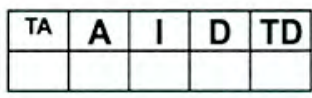

2. La misión de la Facultad concuerda con la de la Universidad.

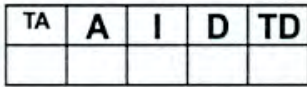

3. El acceso al sistema de información de la Facultad es eficiente y eficaz.

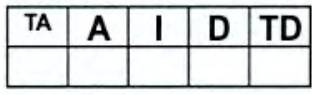

4. La Facultad cuenta con un sistema de comunicación eficiente y eficaz

que facilita la coordinación entre sus distintas áreas y las de la Universidad

5. Los directivos convocan, motivan y dan los lineamientos claros para

lograr los objetivos de la Facultad.
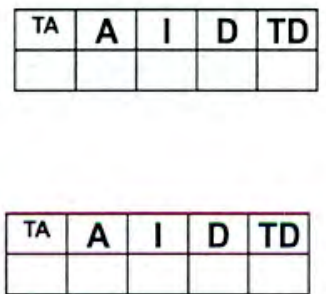

6. La dedicación horaria de los directivos cubre los requerimientos que demanda el personal administrativo de la carrera.
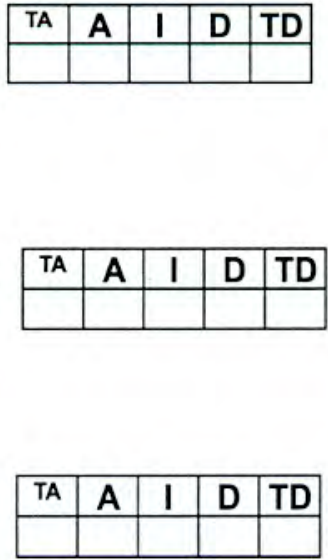
7. Las normas que emite la dirección de la Facultad están claramente definidas.

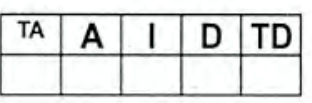

8. ¿Conoce las normas de administración de la Facultad?.

9. Las acciones de motivación e incentivos estimulan al persona administrativo para construir al desarrollo de la institución.

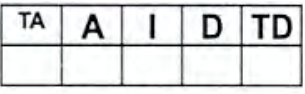

\section{Dimensión 4: Infraestructura y Equipamiento}

1. El servicio de atención de la salud cuenta con locales y equipamiento apropiados

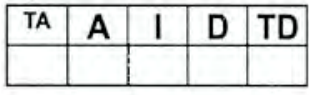

2. Los ambientes de trabajo cuentan con el equipo y mobiliario apropiado para desarrollar con eficacia sus labores administrativas

3. El horario de atención de la biblioteca responde a las necesidades que demandan los usuarios
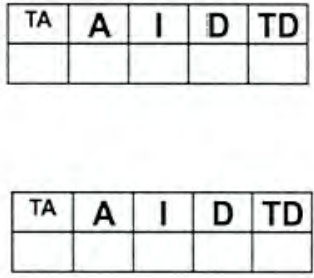

4. La biblioteca cuenta con textos suficientes en numero y calidad temática para

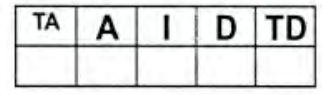

Información Para Procesos de Autoevaluación uso de Instrumentos

\section{Egresados}

Facultad: Año de Ingreso:

Sexo: \begin{tabular}{|l|l|}
\hline M & F \\
\hline
\end{tabular}

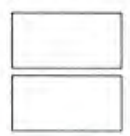

Dimensión 1: La Carrera en el Contexto Institucional

1. El servicio que brinda el personal administrativo es eficiente $y$ eficaz.

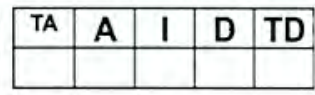

2. El acceso al sistema de información de la Facultad es eficiente y eficaz.

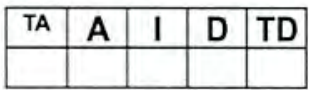

3. La Facultad cuenta con un sistema de comunicación eficiente y eficaz que facilita la coordinación entre sus distintas

\begin{tabular}{|l|l|l|l|l|}
\hline TA & A & I & D & TD \\
\hline & & & & \\
\hline
\end{tabular}
áreas y las de la Universidad

4. Las normas que emite la dirección de la Facultad están claramente definidas.

El perfil del egresado de la carrera está claramente formulado
Dimensión 2: Proyecto Académico

5. El perfil de egresado definido para la carrera responde a los requerimientos exigidos en el

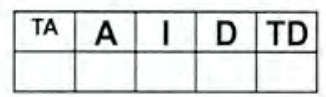
medio laboral.

6. El proceso para la obtención del titulo profesional está claramente formulado.

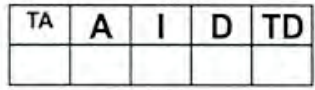

\section{Dimensión 3: Docentes, Alumnos, Egresados}

7. La calidad de la formación profesional recibida guarda correspondencia con las exigencias que demanda el mercado laboral.

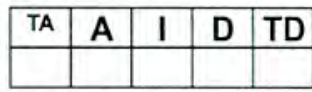

8. El titulo profesional fue necesario para obtener el empleo y condiciones laborales que aspiraba.

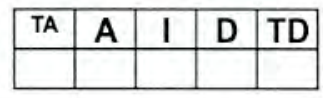

Información para Procesos de Autoevaluación, uso de Instrumentos

\section{Grupos de Interés}

\section{Dimensión 1:}

\section{La Carrera en el Contexto Institucional}

1. El servicio que brinda el personal administrativo es eficiente $y$ eficaz.

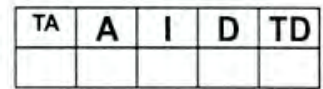

2. El sistema de acceso a la información de la Facultad es eficiente y eficaz.

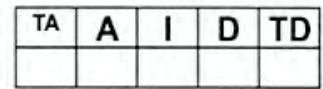

3. La Facultad cuenta con un sistema de comunicación eficiente y eficaz que facilita la coordinación entre sus distintas áreas y las de la Universidad

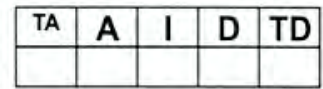

4. Las autoridades de las Facultad muestran disponibilidad para mantener vínculos dentro de su organización

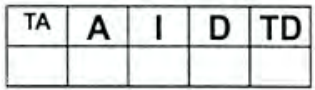




\section{Dimensión 2: Proyecto Académico}

5. Ha tenido la oportunidad de conocer el documento donde se presenta el perfil de egresado.

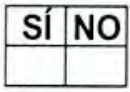

6. El perfil del egresado responde a las requerimientos que demanda su organización.

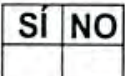

\section{Dimensión 3: Docentes, Alumnos, Egresados}

7. La calidad de la formacion Profesional del egresado guarda correspondencia con La demanda de su organización.

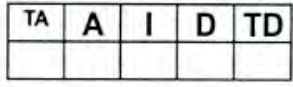

\section{RESULTADO:}

Como consecuencia de la Investigación y con el objetivo de alcanzar la excelencia académica y, por ende, la excelencia profesional del arquitecto egresado de la FAUA, se ha determinado su perfil según competencias.

\section{Perfil del Arquitecto según Competencias}

3.1 Diseñador de proyectos de alta complejidad en contextos nuevos y construidos, con arreglo a la normatividad vigente y que sean sostenibles desde el punto de vista ambiental.

\begin{tabular}{|c|c|c|}
\hline COMPETENCIAS & BASES CONCEPTUALES & BASES PROCEDIMENTALES \\
\hline \multirow{4}{*}{$\begin{array}{l}\text { 1. DISEÑo } \\
\text { Capacidad } \\
\text { para crear } \\
\text { diseños } \\
\text { arquitectónicos } \\
\text { satisfagan } \\
\text { requisitos } \\
\text { técnicos y } \\
\text { estéticos. }\end{array}$} & \multirow{2}{*}{$\begin{array}{l}\text { Capacidad para poner la imaginación } \\
\text { y la creatividad al servicio del } \\
\text { proyecto a fin de invocar y asumir la } \\
\text { dirección de un proceso de diseño. } \\
\end{array}$} & $\begin{array}{l}\text { Capacidad para resolver un proyecto en } \\
\text { forma creativa e innovadora en el } \\
\text { proceso de diseño. }\end{array}$ \\
\hline & & $\begin{array}{l}\text { Habilidad para producir un proyecto de } \\
\text { arquitectura asistido por un programa, } \\
\text { que contemple desde el diseño } \\
\text { eqquemátion hasta el desarrollo detallado } \\
\text { de los espacios programáticos. }\end{array}$ \\
\hline & $\begin{array}{l}\text { Conocimiento de la teoría, los } \\
\text { métodos y los procedimientos } \\
\text { de diseño. }\end{array}$ & $\begin{array}{l}\text { Habilidad para determinar, } \\
\text { seleccionar e integrar los } \\
\text { métodos de diseño y sus } \\
\text { procedimientos. }\end{array}$ \\
\hline & $\begin{array}{l}\text { Conocimientos de los } \\
\text { Antecedentes de diseño y de } \\
\text { critica arquitectónica. }\end{array}$ & $\begin{array}{l}\text { Capacidad para aplicar las } \\
\text { Teorias, los métodos de diseño y las } \\
\text { criticas arquitectónicas al servicio } \\
\text { del proyecto }\end{array}$ \\
\hline $\begin{array}{l}\text { 2. SISTEMAS } \\
\text { DE DISENO: }\end{array}$ & $\begin{array}{l}\text { Conocimiento del urbanismo, } \\
\text { la planificación y las } \\
\text { competencias técnicas que } \\
\text { intervienen en el proceso de } \\
\text { Planificación }\end{array}$ & $\begin{array}{l}\text { Habilidad para evaluar las } \\
\text { caracteristicas del terreno para el } \\
\text { disen̂o. }\end{array}$ \\
\hline $\begin{array}{l}\text { Capacidad de } \\
\text { conciliar } \\
\text { factores } \\
\text { divergentes, } \\
\text { integrándolos a } \\
\text { la concepción y } \\
\text { al desarrollo de } \\
\text { los ambientes } \\
\text { internos y } \\
\text { externos. }\end{array}$ & $\begin{array}{l}\text { Conocimiento de los sistemas } \\
\text { urbanos, así como de } \\
\text { sistemas de transporte, } \\
\text { comunicación, mantenimiento } \\
\text { y seguridad. }\end{array}$ & $\begin{array}{l}\text { Habilidad para determinar } \\
\text { seleccionar e integrar los sistemas } \\
\text { de servicios urbanos, así como de } \\
\text { Transporte, comunicación, } \\
\text { mantenimientos y seguridad en } \\
\text { el diseño arquitectónico. }\end{array}$ \\
\hline $\begin{array}{l}\text { 3. CULTURAY } \\
\text { ARTE: }\end{array}$ & $\begin{array}{l}\text { Conocimiento de la historia y } \\
\text { la práctica de la arquitectura, } \\
\text { el ordenamiento territorial. }\end{array}$ & $\begin{array}{l}\text { Comprensión de asuntos referidos } \\
\text { al patrimonio en el entomo } \\
\text { construido. }\end{array}$ \\
\hline $\begin{array}{l}\text { Capacidad para } \\
\text { actuar con } \\
\text { conocimiento de } \\
\text { los antecedentes } \\
\text { históricos y } \\
\text { Culturales. }\end{array}$ & $\begin{array}{l}\text { Conciencia de los vinculos } \\
\text { existentes entre la arquitectura } \\
\text { y otras disciplinas creativas }\end{array}$ & $\begin{array}{l}\text { Capacidad para actuar con el } \\
\text { conocimiento de las bellas artes } \\
\text { como un factor susceptible de } \\
\text { ejercer influencia sobre la calidad } \\
\text { del diseño arquitectónico }\end{array}$ \\
\hline $\begin{array}{l}\text { 4. SOCIEDAD } \\
\text { Capacidad para } \\
\text { entender, valores, } \\
\text { normas de conducta } \\
\text { y patrones sociales. }\end{array}$ & $\begin{array}{l}\text { Comprensión de los cambios } \\
\text { sociales, politicos, tecnológicos, } \\
\text { ecológicos, y económicos que } \\
\text { definen la practica de la arquitectura }\end{array}$ & $\begin{array}{l}\text { Capacidad para desarrollar un proyecto } \\
\text { mediante la defifinión de las necesidades } \\
\text { de la sociedad, los usuarios y los clientes. } \\
\text { y para investigar y definir las exigencias } \\
\text { contextuales y funcionales para distintos } \\
\text { tipos de entornos construidos }\end{array}$ \\
\hline $\begin{array}{l}\text { 5. MEDIO AMBIENTE } \\
\text { Capacidad para } \\
\text { entender los } \\
\text { principios basicos de } \\
\text { la ecologia. }\end{array}$ & $\begin{array}{l}\text { Conocimiento de las teorias y de los } \\
\text { métodos que buscan esclarecer las } \\
\text { relaciones entre el comportamiento } \\
\text { humano y el medio ambiente. }\end{array}$ & $\begin{array}{l}\text { Capacidad de responder a las } \\
\text { características de medio natural y } \\
\text { construió en el desarrollo de un } \\
\text { programa y el diseño de un proyecto. }\end{array}$ \\
\hline
\end{tabular}

3.2 Investigador capaz de identificar problemas y proponer soluciones arquitectónicas y urbanas.

\begin{tabular}{|c|c|c|}
\hline COMPETENCIAS & $\begin{array}{l}\text { BASES } \\
\text { CONCEPTUALES }\end{array}$ & BASES PROCEDIMENTALES \\
\hline \multirow{3}{*}{$\begin{array}{l}\text { 7. BASES DE } \\
\text { INVESTIGACION: } \\
\text { Capacidad para la } \\
\text { búsqueda y la consulta } \\
\text { sistemática aue } \\
\text { conduzca a la innovación } \\
\text { en el disefo, la la la } \\
\text { planificación, } \\
\text { construcción y la gestión } \\
\text { del entorno construido y } \\
\text { natural. }\end{array}$} & $\begin{array}{l}\text { Conocimiento de las } \\
\text { teorias y de los } \\
\text { métodos de } \\
\text { investigación clentifica. }\end{array}$ & $\begin{array}{l}\text { Habilidad para generar, analizar. } \\
\text { evaluar opciones de diseño y } \\
\text { elaborar conclusiones con rigor } \\
\text { teórico y metodológico. }\end{array}$ \\
\hline & $\begin{array}{l}\text { Conocimiento de los } \\
\text { métodos particulares } \\
\text { necesarios para } \\
\text { elaborar el programa } \\
\text { integral de un proyecto, } \\
\text { incluyendo ia } \\
\text { determinacion de las } \\
\text { necesidades dei cliente } \\
\text { o del usuario. }\end{array}$ & $\begin{array}{l}\text { Habilidad para hacer el exhaustivo } \\
\text { analisis y la evaluación de un } \\
\text { edificio, un complejo de edificios o } \\
\text { del espacio urbano } \\
\text { Capacidad para conceptualizar y } \\
\text { desarrollar los proyectos de } \\
\text { arquitectura y diseñó urbano. }\end{array}$ \\
\hline & $\begin{array}{l}\text { Formación adecuada en } \\
\text { técnicas } \\
\text { investigación como de } \\
\text { parte inherente del } \\
\text { aprendizaje de la } \\
\text { arquitectura }\end{array}$ & $\begin{array}{l}\text { Capacidad para recopila } \\
\text { información, dofinir problemas, } \\
\text { aplicar análisis y juicios criticos, y } \\
\text { formular estratogias de acción y } \\
\text { criterios de valoración, bajo } \\
\text { rigurosos argumentos teóricos. }\end{array}$ \\
\hline
\end{tabular}

3.3 Gestor con competencias para generar, planificar, administrar, controlar y evaluar proyectos de su especialidad.

\begin{tabular}{|c|c|c|}
\hline COMPETENCIAS & $\begin{array}{c}\text { BASES } \\
\text { CONCEPTUALES }\end{array}$ & BASES PROCEDIMENTALES \\
\hline 8. GESTIÓN LEGAL: & $\begin{array}{l}\text { Comprensión de los } \\
\text { códigos; } \\
\text { regulaciones y las las }\end{array}$ & $\begin{array}{l}\text { Habilidad para actuar en el } \\
\text { contexto legal de desarrollo de la } \\
\text { práctica profesional del arquitecto. }\end{array}$ \\
\hline $\begin{array}{lr}\text { Capacidad } & \text { de } \\
\text { entender } & \text { las } \\
\text { responsabilidades } \\
\text { legales del arquitecto. }\end{array}$ & al sitio $\mathrm{y}$ al diseño. & $\begin{array}{l}\text { Conciencia de la importancia del } \\
\text { papel de la documentación técnica } \\
\text { y de las especificaciones en la } \\
\text { realización del disenfo. }\end{array}$ \\
\hline $\begin{array}{l}\text { 9. GESTION } \\
\text { ORGANIZACIONAL } \\
\text { YECONOMICA: }\end{array}$ & $\begin{array}{l}\text { Comprensión de los } \\
\text { principios de negocio y } \\
\text { su aplicación a la } \\
\text { gestión de proyectos. }\end{array}$ & $\begin{array}{l}\text { Capacidad para desarrollar un } \\
\text { proyecto de inversión mediante el } \\
\text { entendimiento y la definición de las } \\
\text { necesidades de la sociedad, los } \\
\text { usuarios y los clientes. }\end{array}$ \\
\hline $\begin{array}{l}\text { Capacidad para actuar } \\
\text { con conocimiento de } \\
\text { los contextos }\end{array}$ & & $\begin{array}{l}\text { Conciencia del papel potencial de } \\
\text { los arquitectos en áreas de } \\
\text { actividad tanto tradicionales como } \\
\text { nuevas, así como en un contexto } \\
\text { internacional }\end{array}$ \\
\hline $\begin{array}{l}\text { Profesionales, } \\
\text { comerciales, } \\
\text { financieros y juridicos, } \\
\text { y para resolver los } \\
\text { aspectos de } \\
\text { financiación y gestión } \\
\text { de proyectos }\end{array}$ & $\begin{array}{l}\text { Comprensión de los } \\
\text { fundamentos } \\
\text { financieros } \\
\text { económicos, y del y } \\
\text { control de costos de la } \\
\text { construcción aplicables } \\
\text { al diseño del proyecto } \\
\end{array}$ & $\begin{array}{l}\text { Habilidad para elaborar MetradOs y } \\
\text { presupuestos de obra } \\
\text { Capacidad de aplicar los sistemas } \\
\text { de evaluación por medios } \\
\text { manuales y/o electrónicos, con el } \\
\text { fin de valorar el rendimiento de los } \\
\text { entornos construidos } \\
\end{array}$ \\
\hline
\end{tabular}

3.4 Ejecutor de obras con dominio de tecnologias en sistemas arquitectónicos, ambientales, constructivos, estructurales , urbanos y afines.

\begin{tabular}{|c|c|c|}
\hline COMPETENCIAS & $\begin{array}{c}\text { BASES } \\
\text { CONCEPTUALES }\end{array}$ & BASES PROCEDIMENTALES \\
\hline \multirow{4}{*}{$\begin{array}{l}\text { Capacidad de } \\
\text { intervenir } \\
\text { utilizando las } \\
\text { competencias } \\
\text { en materia de } \\
\text { las técnicas de } \\
\text { construcción, y } \\
\text { comprensión de } \\
\text { su evolución }\end{array}$} & $\begin{array}{l}\text { Desarrollo de una } \\
\text { competencia creativa } \\
\text { en materia de técnicas } \\
\text { constructivas. }\end{array}$ & $\begin{array}{l}\text { Capacidad de innovación en } \\
\text { tecnologias de construcción. }\end{array}$ \\
\hline & $\begin{array}{l}\text { Conocimiento de } \\
\text { materiales, técnicas y } \\
\text { procedimientos } \\
\text { constructivos, } \\
\text { estructurales. }\end{array}$ & $\begin{array}{l}\text { Habilidad para aplicar } \\
\text { materiales, técnicas y } \\
\text { procedimientos a la ejecución } \\
\text { de obras }\end{array}$ \\
\hline & $\begin{array}{l}\text { Comprensión la labor } \\
\text { regente de los } \\
\text { arquitectos en el } \\
\text { proceso constructivo. }\end{array}$ & $\begin{array}{l}\text { Habilidad para el manejo de } \\
\text { recursos humanos, su ambiente } \\
\text { laboral y el manejo financiero } \\
\text { eficiente. }\end{array}$ \\
\hline & $\begin{array}{l}\text { Conocimiento de los } \\
\text { principios, normas y } \\
\text { procedimientos del } \\
\text { sector de la } \\
\text { construcción nacional } \\
\text { e internacional, publico } \\
\text { y privado }\end{array}$ & $\begin{array}{l}\text { Conciencia del papel del sector } \\
\text { de la construcción en la } \\
\text { economia Nacional e } \\
\text { Internacional } \\
\text { Habilidad para diferenciar los } \\
\text { procedimientos referidos al } \\
\text { acceso a licitaciones y } \\
\text { concursos nacionales e } \\
\text { internacionales, públicos o } \\
\text { privados. }\end{array}$ \\
\hline
\end{tabular}


3.5 Humanista sensible a las diversas formas de expresión, cultural, capaz de entender y adaptarse a las necesidades sociales.

\begin{tabular}{|c|c|c|}
\hline COMPETENCIAS & BASES CONCEPTUALES & BASES PROCEDIMENTALES \\
\hline \multirow{2}{*}{$\begin{array}{l}11 \text { ETICA } \\
\text { Dominio de los } \\
\text { aspectos êticos } \\
\text { para la práctica del } \\
\text { diseño y de la } \\
\text { arquitectura. }\end{array}$} & \multirow[t]{2}{*}{$\begin{array}{l}\text { Conocimientos de flosofia, } \\
\text { politica y ética ascciados a } \\
\text { la labor del arquitecto. }\end{array}$} & $\begin{array}{l}\text { Capacidad para actuar sobre la base } \\
\text { de principios de ética professional. }\end{array}$ \\
\hline & & $\begin{array}{l}\text { Capacidad para actuar con } \\
\text { responsabilidad y compromiso } \\
\text { respecto a la conservación del } \\
\text { ambiente y de los recursos naturales. }\end{array}$ \\
\hline $\begin{array}{l}12 \text { IDENTIDAD } \\
\text { Capacidad de } \\
\text { comprender la } \\
\text { realidad nacional } \\
\text { para preservar la } \\
\text { diversidad cultural }\end{array}$ & $\begin{array}{l}\text { Conocimiento de la } \\
\text { responsabilidad ante los } \\
\text { valores humanos, sociales, } \\
\text { culturales. urbanos. } \\
\text { arquitectónicos y } \\
\text { medioambientales y ante el } \\
\text { patrimonio arquitectónico } \\
\text { nacional. }\end{array}$ & $\begin{array}{l}\text { Capacidad de entender las } \\
\text { tradiciones nacionales y la herencia } \\
\text { regional y local en diseño } \\
\text { arquitectónico, urbano y paisaijstico, } \\
\text { incluidas las costumbres vernáculares }\end{array}$ \\
\hline \multirow{2}{*}{$\begin{array}{l}\text { 1.3. ADAPTACIÓN } \\
\text { Capacidad para } \\
\text { adaptarse } \\
\text { desarrollo } \\
\text { científico, } \\
\text { tecnológico } \\
\text { social } \\
\text { promoverlo } \\
\text { mediante } \\
\text { innovación. } \\
\end{array}$} & \multirow{2}{*}{$\begin{array}{l}\text { Conocimiento de las } \\
\text { tendencias cientificas, } \\
\text { tecnológicas y sociales, en } \\
\text { el àmbito local y } \\
\text { globalizado. }\end{array}$} & $\begin{array}{l}\text { Actitud proactiva para adajtarse a los } \\
\text { cambios cientificos, tecnológicos y } \\
\text { sociales }\end{array}$ \\
\hline & & $\begin{array}{l}\text { Habilidad para involucrarse en } \\
\text { procesos de innovación que buscan } \\
\text { elevar la calidad de vida de la } \\
\text { población. }\end{array}$ \\
\hline
\end{tabular}

\section{CONCLUSIONES}

1. El tiempo empleado en el proceso de investigación del proyecto planteado fue insuficiente para alcanzar las metas trazadas.

2. Investigar sobre excelencia académica como procedente de la excelencia profesional implica una investigación minuciosa y responsable sobre los procesos de autoevaluación, evaluación externa y acreditación.
3. Estando las universidades peruanas, publicas y privadas retrasadas en el proceso latinoamericano y mundial de acreditación, es imprescindible impulsar este proceso para cambiar el estatus actual.

4. La Asamblea Nacional de Rectores ha impulsado la formación de la comisión de acreditación de universidades públicas y privadas.

5. El grupo de trabajo de CRI-SUR ha planteado lineamientos para la acreditación de las facultades de arquitectura, por lo que se debe tomar esta base informativa para iniciar el proceso de autoevaluación y acreditación de la FAUA-UNJBG

6. La investigación académica sobre excelencia académica y excelencia profesional requiere de un tiempo mayor a un año académico, por lo que debe replantearse el plazo propuesto.

\section{RECOMEDACIONES}

- La Asamblea Universitaria de la Universidad Nacional Jorge Basadre Grohmann debe plantearse, como tema imprescindible e impostergable de gobierno, el proceso de autoevaluación y acreditación de todas las Facultades.

- El Consejo de Facultad de la Facultad de Arquitectura, Urbanismo y Artes debe plantearse como meta de gobierno la autoevaluación para la acreditación nacional e internacional nominando una comisión especial permanente por un periodo mayor a un año académico. 